\title{
Preliminary Results from the AFRL-NASA W/V-Band Terrestrial Link Experiment in Albuquerque, NM
}

\author{
Michael Zemba, James Nessel, Jacquelynne Houts \\ Advanced High Frequency Branch \\ NASA Glenn Research Center \\ Cleveland, OH, USA
}

\begin{abstract}
Atmospheric propagation models and the measurements that train them are critical to the design of efficient and effective space-ground links. As communication systems advance to higher frequencies in search of higher data rates and open spectrum, a lack data at these frequencies necessitates new measurements to properly develop, validate, and refine the models used for link budgeting and system design. In collaboration with the Air Force Research Laboratory (AFRL), NASA Glenn Research Center has deployed the W/V-band Terrestrial Link Experiment (WTLE) in Albuquerque, NM to conduct a measurement campaign at 72 and $84 \mathrm{GHz}$, among the first atmospheric propagation measurements at these frequencies. WTLE has been operational since October 1, 2015, and the system design shall be herein discussed alongside preliminary results and performance.
\end{abstract}

Keywords-millimeter wave propagation, propagation losses, radiowave propagation.

\section{INTRODUCTION}

While NASA's communications architectures are currently moving toward $\mathrm{Ka}$ and optical frequencies, the agency is also investigating available spectrum in the $\mathrm{Q} / \mathrm{V} / \mathrm{W}$-bands as a downlink option for the next generation Space Based Relay (SBR) to supercede the Tracking and Data Relay Satellite (TDRS) system in coming years [1]. Accordingly, NASA Glenn Research Center (GRC) and the Air Force Research Laboratory (AFRL) have initiated a joint terrestrial propagation campaign to characterize rain attenuation, depolarization, scintillation, and gaseous absorption effects of the atmosphere in the $\mathrm{V}$ and $\mathrm{W}$ bands (72 GHz and $84 \mathrm{GHz}$ ). The W/V-Band Terrestrial Link Experiment (WTLE) consists of a transmitter on the crest of the Sandia Mountains and a receiver on the roof of a University of New Mexico research center (COSMIAC) in south Albuquerque. The slant path is approximately $24 \mathrm{~km}$ long, with the receiver and transmitter at elevations of $1.6 \mathrm{~km}$ and $3.2 \mathrm{~km}$ respectively, resulting in a look angle of $3.9^{\circ}$ above the horizon. Fig. 1 shows the location of the transmitter at Sandia Crest, the receivers on the roof of the COSMIAC building, and an elevation profile of the slant path covered by the link. The link was installed in late September 2015 and has been operational since October 1, 2015.

\section{SYSTEM DESIGN}

The WTLE transmitter consists of a coherent 72 and $84 \mathrm{GHz}$ continuous wave $(\mathrm{CW})$ beacon with an EIRP of $40 \mathrm{dBm}$ using two lens antennas with a $3^{\circ}$ half-power beam-width and $35 \mathrm{dBi}$

\author{
Nicholas Tarasenko, Steven Lane, David Murrell \\ Space Vehicles Directorate \\ Air Force Research Laboratory, Kirtland AFB \\ Albuquerque, NM, USA
}

directivity. The transmitter, including the lens antennas, is contained in a weather-proof enclosure with the electronics temperature controlled to within $\pm 0.01^{\circ} \mathrm{C}$ and air temperature controlled to within $\pm 0.1^{\circ} \mathrm{C}$. Polarization is LHCP from the perspective of the receivers.

The receiver system consists of two $0.5 \mathrm{~m} \mathrm{~V}$ and $\mathrm{W}$-band Cassegrain reflectors. The twin receivers observe both the coand cross-polarization components of each channel and downconvert the signals to $7 \mathrm{MHz}$ at the feed before digitization. The receiver electronics are also temperature controlled to within $\pm 0.01^{\circ} \mathrm{C}$, and a calibration tone is injected before the LNA for use in periodically monitoring overall system gain. Dynamic range, isolation, and other specifications of the receiver are summarized in Table 1. After digitization, the signals are processed using a Fast Fourier Transform (FFT) I/Q algorithm utilized in previous NASA propagation terminals [2] which uses a frequency estimation technique to coherently track and measure the amplitude of the beacon signals [3, 4]. An overall measurement rate of $10 \mathrm{~Hz}$ rate is implemented to characterize scintillation effects. The receive site also has a variety of colocated meteorology equipment including a weather station, laser disdrometer, and SODAR. Weather instrumentation is also planned for installation at the transmit site and at an intermediate location along the path. More information on the experiment design and instrumentation is presented in [5].
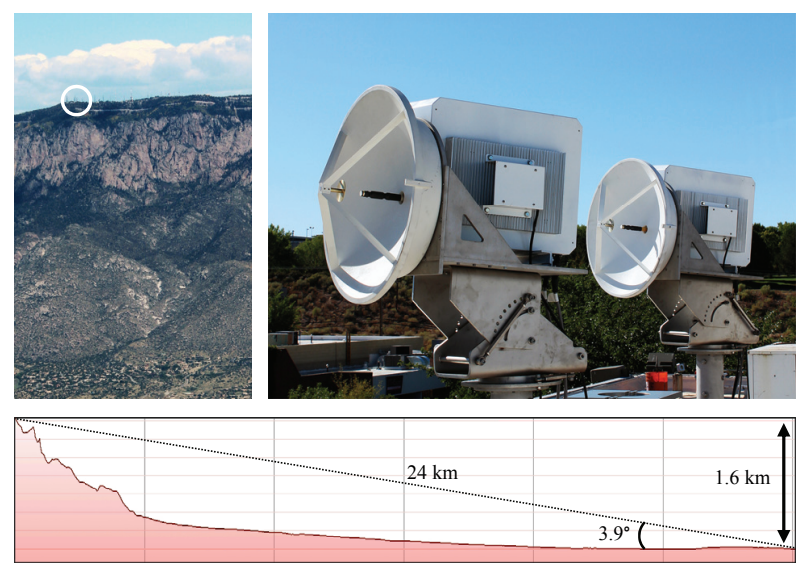

Fig. 1. The location of the transmitter atop Sandia Crest (left); the V and W-band receivers in Albuquerque, NM (right); and the elevation profile of the slant path covered by the link (bottom). 
TABLE I. ReCEIVER Specifications

\begin{tabular}{|c|c|c|}
\hline Channel & Parameter & Spec. \\
\hline \multirow{2}{*}{$\begin{array}{c}V \\
(72 \mathrm{GHz})\end{array}$} & Dynamic Range & $70 \mathrm{~dB}$ \\
\hline & $\mathrm{Co} /$ Cross-Polarization Isolation & $13 \mathrm{~dB}$ \\
\hline \multirow{4}{*}{$\begin{array}{c}W \\
(84 \mathrm{GHz})\end{array}$} & Dynamic Range & $68 \mathrm{~dB}$ \\
\hline & $\mathrm{Co} /$ Cross-Polarization Isolation & $20 \mathrm{~dB}$ \\
\hline & Polarization & LHCP \\
\hline & Measurement Rate & $10 \mathrm{~Hz}$ \\
\hline
\end{tabular}

\section{PRELIMINARY RESULTS}

The link has not yet been operational long enough to analyze long term results with statistical significance, however, several notable examples of various weather phenomena have been observed and are presented here as preliminary findings to demonstrate system performance. Figs. $3-5$ plot the time series power measurements of the co- and cross-polarization channels at both $\mathrm{V}(72 \mathrm{GHz})$ and $\mathrm{W}(84 \mathrm{GHz})$ for various weather events. During clear sky conditions, the received power levels are approximately $-14 \mathrm{dBm}$ (co) and $-27 \mathrm{dBm}$ (cross) for the Vband, and $-17 \mathrm{dBm}$ (co) and $-37 \mathrm{dBm}$ (cross) for the W-band.

Firstly, an example of a deep rain fade event is presented in Fig. 3. For approximately 24 minutes during the event, the measured attenuation exceeds the system dynamic range of 70 $\mathrm{dB}$ on all channels. The concurrent rain rate measured at the receiver site peaked at $17.2 \mathrm{~mm} / \mathrm{hr}$, during this time, but may have been stronger along the path given the severe attenuation. No significant depolarization was observed throughout the event. However, in Fig. 4, a snow event is presented in which strong depolarization effects were measured. In fact, depolarization was observed to the extent that the W-band crosspolarization power level exceeded its respective co-polarization power during the height of the event. This event corresponds to a cumulative snowfall of $7.6 \mathrm{~mm}$ in Albuquerque throughout the day. Lastly, Fig. 5 presents an example of observed cloud attenuation over a period of 6 hours. The WTLE system is uniquely situated to measure cloud effects given that clouds often form along the path. As clouds pass along the link throughout the event in Fig. 5, attenuation rapidly fluctuates by up to $12 \mathrm{~dB}(\mathrm{~V})$ and $15 \mathrm{~dB}(\mathrm{~W})$, while the isolation between the co- and cross-polarizations remains consistent on both channels.

\section{CONCLUSIONS}

Herein, we presented preliminary results from the joint NASA-AFRL terrestrial link experiment at W/V-band, which has been operating in Albuquerque since October 2015. The system has thus far performed as expected and recorded reliable data, including several examples of deep rain fades, depolarization effects, and cloud attenuation, which will ultimately be used to validate theoretical models of atmospheric propagation phenomena at $\mathrm{V}$ and $\mathrm{W}$ band. Future work for this campaign include the installation of additional weather instrumentation at the transmitter site and along the slant path, as well as a more thorough analysis of the collected data once the system has been operational for a statistically significant length of time.

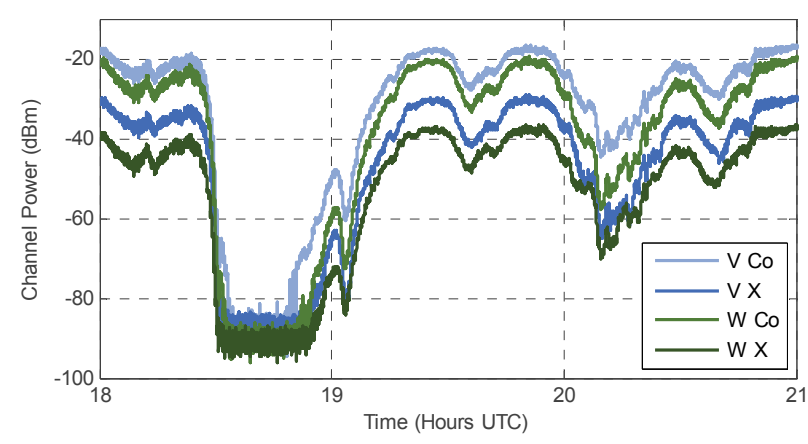

Fig. 3. Time series of received power during a deep rain fade on November $4^{\text {th }}, 2015$. Total attenuation exceeds the receiver dynamic range on all channels for approximately 24 minutes from 18:31 - 18:55 UTC.

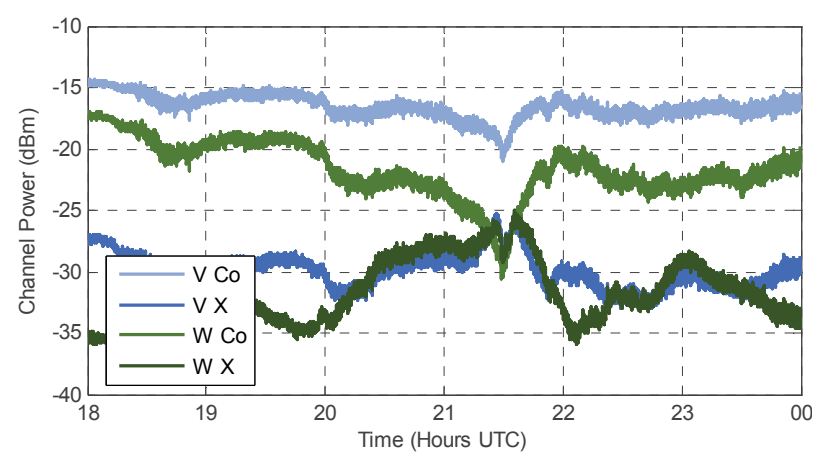

Fig. 4. Time series of received power levels during a snow event on December $26^{\text {th }}, 2015$ showing strong depolarization effects during the height of the event at approximately 21:30 UTC.

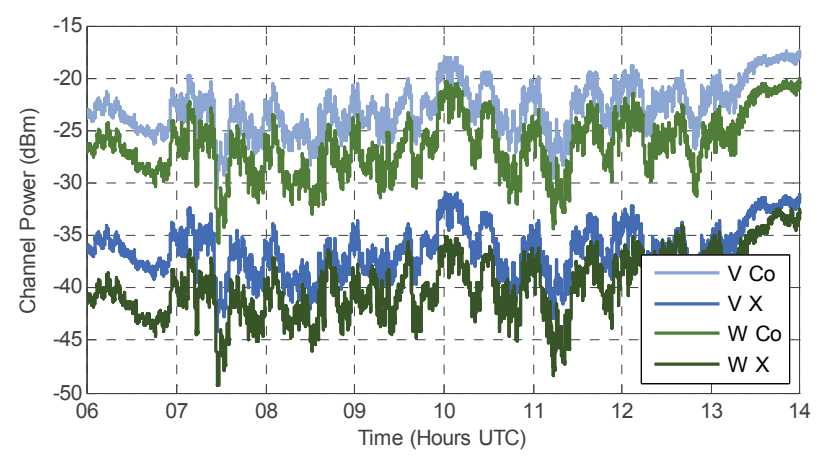

Fig. 5. Time series of received power levels during a cloud event on January $9^{\text {th }}, 2016$. From approximately 07:00 - 13:15 UTC, clouds passed along the link introducing variations in attenuation up to $15 \mathrm{~dB}$.

\section{REFERENCES}

[1] Space Communications and Navigation Office, "NASA's Space-Based Relay Study: Overview and Direction," Washington, DC: March 2013.

[2] R. Acosta, B. Frantz, J. Nessel, "Goldstone Site Test Interferometer," $13^{\text {th }} \mathrm{Ka}$ and Broadband Communications Conference, Turin, Italy, 2007.

[3] "Software-Defined Beacon Receiver Implementation Using Frequency Estimation Algorithms," NASA Technology Briefs, Vol. 39 No. 6, pp. 58-59: LEW-19222-1.

[4] J. Nessel, M. Zemba, J. Morse, "Design of a K/Q-band Beacon Receiver for the Alphasat TDP\#5 Experiment," 2014 IEEE Int. Symp. on Antennas and Propag., Memphis, TN. July 6-11 2014.

[5] N. Tarasenko, S. Lane, D. Murrel, J. Nessel, M. Zemba, J. Houts, "W/VBand Terrestrial Link Experiment, an Overview," 2016 IEEE Int. Symp. on Antennas and Propag., Fajardo, Puerto Rico. June 26 - July 1, 2016, in press. 